\title{
Le poids militaire du Sud : le grand désordre
}

\section{Marie-Lucy Dumas}

\section{(2) OpenEdition}

1 Journals

\section{Édition électronique}

URL : http://journals.openedition.org/conflits/88

DOI : $10.4000 /$ conflits.88

ISSN : 1777-5345

Éditeur :

CCLS - Centre d'études sur les conflits lilberté et sécurité, L'Harmattan

\section{Édition imprimée}

Date de publication : 17 mai 1991

ISSN : 1157-996X

\section{Référence électronique}

Marie-Lucy Dumas, "Le poids militaire du Sud : le grand désordre », Cultures \& Conflits [En ligne], 02 | printemps 1991, mis en ligne le 30 décembre 2002, consulté le 30 mars 2021. URL : http://

journals.openedition.org/conflits/88; DOI : https://doi.org/10.4000/conflits.88

Ce document a été généré automatiquement le 30 mars 2021.

Creative Commons License 


\title{
Le poids militaire du Sud : le grand désordre
}

\author{
Marie-Lucy Dumas
}

"Il ne reste qu'une génération pour séparer l'Occident de l'entrée en scène du tiers monde" : cette confidence de De Gaulle à Malraux, rapportée dans Les chênes qu'on abat, est-elle en train de se vérifier? C'est la question qu'on peut se poser après l'effondrement de l'Union soviétique et l'alerte irakienne alors que certains n'hésitent pas à faire une sorte de transfert de la "menace de l'Est" à la "menace du Sud", Sud où se situeraient désormais tous les dangers.

Un passé récent invite, toutefois, à la prudence. Dans la décennie 70, plusieurs auteurs éminents comme Stanley Hoffman, Jacques Lesourne ou Pierre Mayer ${ }^{1}$ avaient, en effet, souligné que la maîtrise de la société internationale devenant de plus en plus complexe, elle tendait à s'éparpiller, bref que les deux grands n'étaient plus ce qu'ils étaient et que l'on passait non seulement à un bipolarisme atténué, mais à un certain polycentrisme. Or, quinze ans après, on attend toujours l'émergence, comme nouveaux pôles de puissance de l'Inde, de la Chine, du Mexique ou du Brésil qu'en fait la crise économique mondiale et les effets pervers d'un développement mal calculé ont freiné au point d'élargir même parfois le fossé qu'ils ambitionnaient une fois pour toute de franchir. On dira qu'il s'agit ici de parler non point de poids économique, mais de poids militaire, et qu'à l'évidence l'acquisition d'un arsenal semble plus facile à réaliser qu'un véritable développement, la peur, l'ambition et la volonté de puissance s'avérant de surcroît des motifs d'action souvent plus déterminants que celui d'améliorer le bienêtre général. Beau sujet de discussion entre puissance militaire et puissance économique, qui, au-delà du triptyque classique développement - sécurité désarmement, soulève bien des questions : peut-on devenir durablement une puissance militaire sans être une puissance économique (problème des pays du Sud), peut-on rester une puissance militaire quand l'économie flanche (problème des états-Unis), enfin peut-on demeurer une grande puissance économique sans tôt ou tard se doter d'un armement à la mesure du rang industriel et financier auquel on a, par ailleurs, accédé (problème de l'Allemagne et du Japon)? 
Pour s'en tenir aux problèmes du Sud, disons que la capacité militaire dépend d'autant plus aujourd'hui de la capacité économique que - comme la guerre du Golfe l'a montré la stratégie moderne implique une telle dimension technologique, une telle intégration électronique de tout l'appareil guerrier que quiconque n'est pas au sommet, perd en réalité toutes ses chances.

Voilà donc qui jette un doute sur l'idée qu'un nouveau partage de puissance est en train de s'effectuer, une nouvelle géopolitique en train de naître: est-ce pour rien, alors qu'on s'attendait à voir la fin de la bipolarisation déboucher sur une sorte de multipolarité, qu'on assiste en définitive avec les Etats-Unis à l'émergence d'un unicentrisme? Non, de ce point de vue, le tiers-monde, en bloc et en détail n'est pas, cette fois encore, très bien parti et, sous bénéfice d'inventaire, ne paraît pas, a priori, prêt à rivaliser! D'autant que, comme le souligne Edward Luttwak ${ }^{2}$, les impératifs nationaux et internationaux, les besoins vitaux et les forces en présence sont telles que la géo-économie risque de plus en plus de prendre le pas sur la géostratégie. Bref, on en arrivera peut-être au désordre mondial dont Alain Joxe a récemment brossé le paysage ${ }^{3}$, mais non à un face à face inexorable. Suggérer le contraire n'est-ce pas, d'ailleurs, sacrifier à une dichotomie élémentaire susceptible au demeurant de susciter au Sud le sentiment qu'une menace du Nord se profile ? S'il existe une géopolitique des armements, et une autre de l'instabilité, dont l'hétérogénéité Nord-Sud accroît les risques, on ne saurait pour autant simplifier. Le Sud est une image facile qui a remplacé le terme "tiers-monde" depuis que l'Union soviétique flirte avec ses anciens ennemis et que la phraséologie non alignée a périclité avec le Mouvement. Le besoin bien humain de classifier pour simplifier, de simplifier pour frapper les esprits, de frapper les esprits pour mobiliser ou désigner la menace nouvelle ou les responsables de la crise intérieure, s'appuie sur encore moins de réalités qu'à l'époque du tiers-mondisme triomphant.

Un Sud ? Non des Sud! Sans régulation - d'où viendrait-elle ?-, sans centre de gravité quelle puissance pourrait ainsi jouer ce rôle? Sans idéologie mobilisatrice face à la concurrence des religions et des particularismes, sans intérêts communs, les 130 états du Sud ne sont qu'un conglomérat de forces disparates, des dragons asiatiques aux PMA africains. Si des ensembles régionaux existent (ASEAN) ou s'esquissent (UMA), ils s'ignorent ou s'opposent plus souvent qu'ils ne s'emboîtent. Qu'on pense à l'effondrement des cours du cacao ou du café provoqué par une concurrence effrénée entre producteurs d'Afrique et d'Amérique latine, concurrence qui a balayé toutes les velléités de régulation des marchés souhaitées, pourtant, par les associations de producteurs créées dans la foulée des succès de l'OPEP.

Le Sud est-il d'ail1eurs aussi séparé et séparable du Nord qu'on le dit. Chaque Etat du Maghreb, par exemple, entretient beaucoup plus de relations avec la CEE qu'avec ses voisins. Sa seule unité, en tout cas, ne pourrait résulter que d'un impérialisme nordiste visant sous prétexte de contrôle, à instaurer un nouvel ordre mondial à sens unique. C'est dire si la recherche de ce dernier et l'établissement d'une nouvelle géopolitique requièrent d'être circonspect.

Géopolitique des armements

Même si l'annuaire 1991 du SIPRI confirme une diminution des dépenses d'armement dans le monde depuis 1987 et une stabilisation dans le tiers-monde, même s'il en va de même, à l'heure du désarmement de Vienne, pour la production et le commerce des armes conventionnelles, il reste que sur ce triple plan des exportations, de l'industrie et 
des budgets militaires, le poids du Sud s'est accru depuis vingt ans, bien plus que le produit national brut. Nombre de facteurs négatifs aggravent bien entendu ce phénomène. En premier lieu, l'entreprise du désarmement rend disponible quantités d'armes qui, comme le montre la cession de chars à l'Egypte ou à la Syrie, peuvent être vendues à bas prix à des pays trop souvent prêts à entasser, comme l'Irak, des matériels disparates. La guerre du Golfe, de son côté, a donné un coup d'arrêt aux velléités de certains de toucher au plus vite les dividendes de la paix et a montré combien la disposition d'engins de plus en plus sophistiqués s'imposait. Il ne manque pas, par ailleurs, d'industries d'armement surdimensionnées, comme en France, ce qui, pour des raisons sociales et économiques évidentes, rend leur reconversion problématique. Parallèlement, les fabrications locales s'étendent tandis que les hésitations américaines prouvent que le souci de contrôle et de limitation s'accompagne, avant même d'avoir reçu un début d'exécution, de fournitures nouvelles à ceux que l'on entend favoriser. S'ajoutent, pour contrecarrer les entraves posées à l'acquisition des technologies sensibles, la collaboration croissante entre pays du Sud, le détournement à des fins militaires des entreprises civiles, sans parler du trafic illégal.

- L'accélération des dépenses militaires a fait augmenter la part du tiers-monde de 3,3 $\%$ en 1955 à $16 \%$ en 1980 et $22 \%$ en 1989 . Onze pays y consacrent plus de $10 \%$ de leur PNB : Israël, la Jordanie, Oman, l'Arabie Saoudite, la Syrie, l'Irak, l'Iran, la Mongolie, l'Angola, l'éthiopie et Cuba. Seuls parmi eux l'Arabie et Oman échappent à un endettement considérable. En ajoutant les états qui consacrent entre 5 et $9 \%$ de leur PNB (Bahreïn,

l'Egypte, le Koweït, les émirats, le Yémen, le Pakistan, l'Inde, le Sri

Lanka, la Corée du Nord, Taiwan, la Malaisie et le Nicaragua), on tombe sur la liste à peu près complète des pays qui, en 1988, soit se situent dans une zone conflictuelle (MoyenOrient et Asie du Sud), soit connaissent sur leur sol des conflits internes (Amérique centrale et Afrique), soit se rattachaient hier au camp soviétique. Notons que $78 \%$ des exportations d'armes du Sud leur sont destinées, que cinq sont essentiellement pourvus par l'Union soviétique (Inde, Irak, Corée du Nord, Syrie, Angola auxquels il faut, sur ce plan, ajouter l'Afghanistan et la Libye), cinq par les Etats-Unis (égypte, Arabie, Israël, Taiwan, Pakistan, premiers fournisseurs, par ailleurs, de la Corée du Sud et de la Thaillande), deux par la Chine (Corée du Nord et Iran). L'Europe intervient dans ces fournitures selon des taux variés, dépassant rarement les $30 \%$ pour l'un ou l'autre de ces pays.

- Les nouveaux producteurs d'armes du Sud ont vu, de leur côté, leur commerce passer de 0 à $10 \%$ de 1960 à 1990. Au regard des grands, les effets de la concurrence de la Chine, du Brésil, d'Israël, de l'égypte, de la Corée du Nord et de la République sudafricaine a été d'autant plus ressentie qu'elle se conjuguait avec une baisse des achats due à la fois à la diminution des revenus pétroliers et à la crise économique. De là, une vaste mutation par concentration, coopération et accroissement de la part civile dans la production aéronautique et électronique, notamment, des industries du Nord.

Sept pays possèdent une capacité à peu près complète de fabrication d'armes conventionnelles (chars, avions, navires, armements légers, artillerie). Il s'agit, dans l'ordre, de la Chine, du Brésil, d'Israël, de l'égypte et de la Corée du Nord, enfin de l'Inde, du Pakistan et de la République sud-africaine. Sur les cent principales 
compagnies mondiales, exportatrices, la 58ème et la 94ème dépendent de Tel Aviv, la 69ème de Séoul, la 82ème de Prétoria, la 92ème de Brasilia et la 93ème de New Delhi. Souci d'indépendance, souci industriel guidé par l'espoir de retombées civiles, toutes sont évidemment à la recherche de transferts de technologie. L'exemple de la Turquie, passée de décennie en décennie de l'achat de matériels démodés à la mise sur pied d'usines d'assemblage et de pièces détachées, suivie du développement d'une industrie diversifiée, pour en arriver aujourd'hui à la fabrication de radars et de navires modernes, est symptomatique. Encore faut-il mentionner tout un trafic illégal, non seulement d'armes, mais de détournement de technologie, associés à la reconversion de narco-dollars que la guerre Irak-Iran et celle du Liban ont favorisés, avec la double complicité d'anciens membres du pacte de Varsovie soucieux d'écouler leurs surplus militaires soviétiques, et d'industriels de pays de l'OTAN dont une liste noire établie par Washington à la suite du Golfe vient de désigner la trentaine d'entreprises à la vindicte publique.

- On passe du quantitatif au qualitatif avec les conflits les plus récents (Afghanistan, Golfe, Liban et dans une moindre mesure Tchad) où l'élévation du niveau technologique des armes employées conduit au développement des industries domestiques. Avions de combat sophistiqués, missiles sol-air, anti-navires ou balistiques à courte et moyenne portée, systèmes de surveillance radio, radar et électroniques, armes chimiques toutes les puissances régionales s'efforcent de se procurer ou de fabriquer ces dernières nouveautés! De l'assemblage en kit sous licence, caractéristique du début des années soixante-dix, on en vient à l'émergence de technologies complètes, l'égypte, Israël et l'Iran se dotant, en particulier, d'une électronique performante, aux côtés de l'Inde où 150000 ouvriers travaillent dans l'industrie d'armement. Conjointement apparaît une coopération Sud-Sud : entre Israël, l'Afrique du Sud et Taiwan, ici sur le plan nucléaire et là pour le missile anti-navire Gabriel; entre Israël et la Chine pour un système de guidage balistique. L'Irak l'Argentine et l'égypte ont úuvré en commun au projet Condor. La Corée du Nord améliore les Scud2 soviétiques au bénéfice de la Syrie et de la Libye, et se lie à l'Iran pour construire une usine destinée à cette même fusée. Le Pakistan collabore avec la Chine pour un missile à moyenne portée.

L'Inde a vendu à l'Irak des produits chimiques. Ainsi émergent quelques puissances dont les capacités sont d'ores et déjà dignes d'être soulignées, et dont l'acquisition de transfert de technologie ne va pas sans répercussions à moyen terme sur les industries d'armement du Nord.

Parmi tous ces pays, la Chine mérite une mention spéciale. Elle apparaît, en effet, comme le promoteur-type ${ }^{4}$ de coopération technologique Sud-sud, spécialisée qu'elle est dans deux créneaux: la technologie nucléaire et celle du missile balistique. Seul pays du Sud au Conseil de Sécurité de l'ONU, membre accrédité du club nucléaire, elle se trouve dans la situation hautement confortable d'être des Grands pour la raison et des Petits pour les affaires. Son comportement pose ouvertement le problème des fauteurs de prolifération.

- Le développement des proliférations au niveau supérieur est, dans ce contexte, inévitable. Lors de la dernière conférence sur le TNP, plusieurs états signataires, reflétant tout compte fait les réticences naguère exprimées par la France face à ce traité, ont stigmatisé l'accaparement par un club de grandes puissances de la capacité 
nucléaire militaire. L'argument est simple, voire simpliste : si cette arme empêche la guerre, tous les pays devraient la posséder argument que la ligue arabe, en avril 1990, reprenait à son compte sur un mode moins rassurant en adjurant ses membres d'accéder à l'armement atomique pour équilibrer la puissance nucléaire de Tel Aviv5.

Parmi la vingtaine de pays capables, sans en avoir nécessairement envie, de se doter de la bombe, quatre ou cinq sont suspectés par les Etats-Unis de vouloir la fabriquer: outre l'Irak dont les centrales et sites ont été largement détruits, l'Iran qui achève l'usine nucléaire de Bouchehr et achète un réacteur expérimental à la Chine, la Corée du Nord, rétive à l'égard de l'Agence internationale pour le contrôle de l'énergie atomique, le Pakistan dont l'attitude en ce domaine demeure pour le moins ambiguë, enfin l'Algérie dont on sait qu'elle dispose d'un réacteur grâce à l'aide de Pékin. Si le problème est ancien, puisqu'il y a des années que les Cassandre prévoient une prolifération dévergondée, il a gagné en gravité avec la vulgarisation des missiles balistiques capables de porter au loin une arme qu'il ne suffit pas de posséder si on ne peut valablement, la délivrer. Encore y a-t-il missile et missile. La plupart des pays connus pour en avoir dans leur panoplie ne disposent, en fait, que d'armes à courte portée. L'Inde, la Chine, l'Arabie Saoudite, Israël et Taiwan développent, en revanche, des fusées de technologie sudiste dont l'allonge, supérieure à 1000 kilomètres, peut leur assurer un rôle régional de premier plan. En pensant à l'Irak et à ses Scud bricolés, on peut toutefois se poser des questions de fiabilité. Quant à l'exportation en provenance du Nord, on sait que depuis 1987 les puissances majeures se sont engagées, en vertu du Missile Technology Control Regime (MTCR), à ne vendre, ni des fusées allant au-delà de 300 kilomètres ni les technologies afférentes. II est vrai que les sanctions sont inexistantes et que comme la guerre du Golfe l'a montré, nombre d'industriels sont loin de respecter les règlements.

Reste la prolifération chimique, dans un sens plus préoccupante parce que plus difficile à déceler et à empêcher. Les Etats-Unis ont dernièrement élaboré, à ce titre, une liste de 28 états vis-à-vis desquels sont renforcés les contrôles d'exportation de produits à double usage : 9 en Asie, les plus avancés, 2 en Europe (URSS et Roumanie), 1 en Afrique (l'Afrique du Sud), tous les autres - Israël inclus - se situant au Proche et au MoyenOrient. Par ailleurs, le groupe informel des 20 pays industrialisés, producteurs de matières chimiques (groupe d'Australie) tente d'harmoniser les règles d'exportation, celle-ci étant, en particulier, interdite pour cinquante produits de référence dont la liste a été dûment établie. On sait, cependant, que le problème est compliqué, non seulement par la multiplicité des substances qui peuvent permettre la mise sur pied d'un arsenal en ce domaine, mais par la modestie des coûts. C'est l'argument de l'arme chimique, arme nucléaire du pauvre capable de faire pièce à la dissuasion atomique des nantis. Argument à plus d'un titre illusoire que la guerre du Golfe, toutefois, risque de ne pas avoir détruit.

En fin de compte, le terme de prolifération reste ambigu et par suite controversé. Pour les pays du Sud, il s'agit simplement d'acquérir des technologies que le Nord veut conserver pour pérenniser sa domination, tandis qu'il s'agit bien entendu pour ce dernier de réduire les risques et de les contrôler. La proposition du président George Bush, datant du 29 mai, dernier n'échappe pas plus que les autres à cette contradiction. Contrôle et limitation constituent une démarche incontournable, mais comment obtenir un consensus sur un tel sujet? 
On trouve, certes, quelques exemples consolants : le Brésil et l'Argentine ont enterré la hache de guerre en s'entendant pour ne pas fabriquer d'armes nucléaires, Buenos Aires venant, par ailleurs d'annoncer qu'il renonçait à développer son programme Condor 2 et décidait de transférer les moyens qu'il lui destinait à une coopération spatiale avec Brasilia. Pareille bonne volonté ne semble pas prête, pourtant, de s'étendre à des zones plus troublées.

- Si elle fait ressortir l'émergence d'un certain nombre de puissances régionales et l'existence de foyers de belligérance actifs ou potentiels, l'analyse ci-dessus esquissée ne doit pas faire oublier tout d'abord, qu'une majorité de pays du Sud demeurent relativement désarmés. Il ne s'agit donc pas de verser dans les simplismes auxquels trop de militaires - obnubilés par la menace - ou trop de spécialistes - soviétologues pour commencer - nous ont habitués. Si l'on prend par exemple pour thème la militarisation de l'Afrique, on s'aperçoit qu'au Sud du Sahara, au moins, elle est fort limitée en effectifs, en matériels, comme en dépenses, les seuls pays surarmés l'étant, telle l'éthiopie, en fonction des conflits auxquels ils ont été liés, voire de l'aide soviétique ou cubaine, hier dispensée à profusion et aujourd'hui arrêtée. Les autres états disposent d'unités essentiellement terrestres de quelques milliers ou dizaines de milliers d'hommes, souvent divisées en armée, gendarmerie, police et garde présidentielle pour que la force ne soit pas concentrée et ne donne pas trop de pouvoir aux militaires, bref des unités dotées de matériels généralement modestes, privés de toute infrastructure moderne. L'Afrique du Sud et le Nigeria font exception. Mais toutes deux ont une armée à leur mesure qui ne reflète nullement une recherche de puissance outrée. La première, sans doute nucléaire, représente à elle seule près de 50 $\% d u$ potentiel du continent où elle ne peut, dès lors, manquer de jouer un rôle éminent, le second se sentira tôt ou tard, s'il n'éclate pas, une vocation à combler les vides que constituent à ses côtés des états faiblement peuplés où l'influence française diminuera fatalement avec le temps. L'Afrique subsaharienne est, il est vrai, comme l'Amérique du Sud peut-être, un cas particulier. Ailleurs, tout au long du Rimland qui s'étend de Tanger à Taiwan, les armées non seulement sont d'une autre ampleur, mais sont surtout susceptibles de se doter un jour de fusées, danger qui souligne la nécessité, en Méditerranée notamment, d'une recherche Nord-Sud de sécurité. Sur ces armées il est difficile de porter un jugement. Dans leur quasi-totalité, elles manquent d'une flotte de transport et de forces navales, même si, en ce domaine comme en d'autres, on peut noter quelques progrès ${ }^{6}$. Leurs forces aériennes risquent, de leur côté, de souffrir d'un manque de support électronique global et, par là même, d'être privées de toute efficacité face à un adversaire mieux équipé. Le Golfe est à cet égard illustratif : la supériorité américaine dans tous les compartiments techniques - de la reconnaissance des satellites aux cruise missiles, en passant par les armes anti-radars ou les lanceroquettes multiples - a eu des effets tels qu'elle se passe de commentaires. Encore l'expérience du Golfe et l'étonnante contre-performance de l'Irak ne sont-elles pas uniques. L'armée chinoise ne se révèle guère plus brillante lorsqu'elle tente de donner une leçon à ses voisins dans les calcaires du

Nord-Vietnam. L'armée indienne ne fait pas mieux au Sri Lanka. La guerre Irak/Iran s'éternise faute, semble-t-il, de stratèges de talent. Les guerres arabes contre Israël se terminent en désastre. La Libye se montre incapable d'utiliser l'arsenal qu'elle a amassé à grands frais et, à Ouadi Doum, se laisse misérablement déposséder du camp retranché qu'elle a pourtant hérissé d'équipements modernes par quelques centaines de Toubous 
dotés d'armes anti-chars et de camionnettes légères. Enfin, les forces argentines s'avèrent totalement déficientes face au corps expéditionnaire anglais. On pourra objecter, il est vrai, que les exemples ci-dessus sont disparates et relèvent pour certains, d'une époque dépassée. Quelle est aujourd'hui la capacité d'assimilation des leçons de la guerre du Golfe pour les pays possédant un type d'arsenal à l'irakienne ? La réponse est en partie donnée peut-être par la Chine qui vient de revoir son plan des 4 modernisations en l'axant sur l'alliance industrie civile-industrie d'armement et sur la modernisation plus particulière de l'armée de l'air : escadrons de Sukoi 27 commandés à l'Union soviétique, couverture radar et système fable de communication. Ce forcing est l'úuvre des réformateurs, de ceux qui veulent sortir du carcan du parti, sans pour autant être favorables à une évolution libérale du régime. Reste à savoir si ce type d'aggiornamento peut suffire à donner l'efficacité recherchée à une armée sur laquelle il y avait, hier encore, beaucoup à redire.

Que conclure sinon que la guerre est en train de changer de nature. Au temps où le quantitatif l'emportait, une force moindre pouvait par le courage de ses soldats ou la clairvoyance de ses chefs rivaliser avec un ennemi mieux doté. C'est encore vrai aujourd'hui pour ce qui concerne les guérillas et nombre de conflits limités. Mais la technologie émergente ne laisse guère de chances à qui est en infériorité : il devient aveugle et bientôt muet. Comme les joueurs de tennis, les armées se classent ainsi, de plus en plus par catégories : qui n'est pas du niveau risque très vite d'être asphyxié et au premier tour éliminé. Le Sud, à cet égard, souffre d'un handicap que sa faiblesse économique lui permettra difficilement de remonter.

Ressortent néanmoins quelques pôles de puissance. Non pas l'égypte, trop pauvre, l'Arabie, trop vide, ou Israël trop petit, mais l'Inde, la Chine, l'Iran, l'Irak hier et peutêtre demain, le Brésil, autant de pays où le niveau d'armement correspond à des capacités économiques et démographiques suffisantes. L'Inde est un bon exemple : elle est volontariste, maintient un outil militaire significatif, a choisi depuis une décennie de s'ancrer comme une puissance régionale éminente capable de contrôler l'océan Indien avec sa flotte ${ }^{7}$ pour qu'il ne devienne pas un "lac américain", capable de tenir en respect le Pakistan avec son armée, de tenir à distance avec ses missiles la Chine, enfin soucieuse, après s'être dotée de l'arme atomique, de prendre place dans l'espace le plus vite possible. Outre, cependant, que le repli sur elle-même de l'URSS lui a fait perdre un soutien, notamment maritime, important, New Delhi s'interroge d'autant plus sur le choix prioritaire à effectuer entre le développement et la sécurité que si cette dernière est à la base de son rôle international, le développement, quant à lui, est indispensable à sa cohérence interne toujours plus ou moins menacée. Facteur fondamental pour l'Inde comme pour tout pays qui prétend à la puissance! Celle-ci dépend, en effet, non seulement du cadre spatial, des ressources et des capacités, qu'elles soient industrielles ou militaires, mais elle dépend aussi de l'organisation sociale et de ce que Raymond Aron appelle la capacité d'action collective, reflet d'un minimum d'équilibre, de consensus et de stabilité. Nouvelle faiblesse que bien des pays du Sud - fussent-ils surarmés - ne sont pas près de pallier.

Géopolitique de l'instabilité

Il n'y a pas que l'armement pour déterminer le poids militaire du Sud et, par suite, influer sur ses relations avec le Nord : il y a, à plus d'un titre, son instabilité. Celle-ci, en effet, est non seulement l'un des facteurs qui intervient dans la militarisation du tiers 
monde, mais c'est aussi, à divers niveaux, une source d'interférences, une cause d'imbrications et de rivalités Nord-Sud qu'il convient de prendre en compte.

- Première considération, les liens entre armements et instabilité, ou si l'on préfère armements et conflits. Ces liens, qui font penser au diptyque désarmementdéveloppement, ne sont pas toujours évidents. Certes, les conflits se déclenchent avec des armes et se nourrissent d'elles, sous peine de très vite s'arrêter, comme ce fut le cas de la "Guerre de Noël" entre le Mali et le Burkina. A cet égard, les grands pays fournisseurs, quelles que soient les bonnes raisons qu'ils peuvent avancer, ont des responsabilités majeures dans les guerres importantes du type Kippour, Irak-Iran, ou Afghanistan, la quantité d'armes nécessaire étant telle que, sans eux, le combat cesserait très vite faute de combattants. On ne saurait, toutefois, oublier que les petits flux d'armements en provenance de quelques pays sudistes (Chine, Corée du Nord, Irak, Israël, Libye) alimentent des conflits internes de moindre ampleur en Afrique ou en Asie, conflits d'une taille suffisante, cependant, pour inciter les producteurs d'armes à penser que le commerce de celles-ci rapporte toujours Une des raisons de l'arrogance khmer rouge dans les négociations tient à la certitude de la livraison de matériel chinois qui est de l'initiative des armées ou des industries sans contrôle gouvernemental. Le Premier ministre Gandhi avait fixé, de son côté, à un milliard de dollars par an ce que devrait rapporter à l'Inde les exportations d'armement en 1990, dans le but de financer l'approvisionnement des armées et d'obtenir un poste excédentaire dans la balance commerciale. L'objectif atteint s'est limité à 600 millions, récoltés néanmoins, à n'en pas douter, dans le sud-est asiatique et les états insulaires de l'océan Indien. Les petits vendeurs auraient-ils moins de scrupules que les cinq ou six gros Européens et Américains? Pour investir des marchés avec des pratiques qui relèvent purement du commerce traditionnel, ils bénéficient de trois préjugés favorables: moins chers, moins contraignants quant aux interdictions de réexportation, enfin et surtout, dépourvus de conditionnalité politique, donc à l'abri des pressions et des embargos.

On ne saurait pour autant simplifier, l'analyse prouvant, en particulier, que l'idée du commerce des armes "initiateur de conflits" est loin, dans la plupart des cas, d'être vérifiée ${ }^{8}$. En fait, pas plus qu'il n'y a la guerre parce qu'il y a des militaires, voire des régimes militaires, il n'y a la guerre parce qu'il y a des armes. Les conflits vivent par eux-mêmes, à telle enseigne que comme le montrent les récents accords de Vienne - il faut généralement attendre qu'ils se résorbent pour désarmer et non l'inverse. Le plan français de contrôle des armements du 3 juin 1991 ne suggère d'ailleurs rien d'autre lorsqu'il souligne que le préalable à tout arrangement régional de sécurité réside dans l'établissement de mesures de confiance et la recherche négociée d'un équilibre entre les parties. Si la disposition par un pays d'un arsenal important n'est donc pas forcément synonyme de danger pour ses voisins pas plus qu'à l'inverse le vide d'armes n'attire automatiquement l'invasion, il reste qu'armement et conflit vont généralement de pair. Qu'on pense à l'URSS armant l'égypte ou exploitant conjointement la décolonisation portugaise et la révolution éthiopienne! Son vecteur est la fourniture ou la vente d'armes tous azimuts, sa stratégie la déstabilisation du continent. Le résultat est à la fois le durcissement et l'extension des conflits en même temps que leur internationalisation sous le coup de la rivalité des deux Grands. L'Afrique devient un enjeu stratégique. Son poids militaire s'accroît d'autant! On retrouve un phénomène voisin au Proche-Orient avec le cliquetis permanent des armes, l'hypertrophie des 
budgets et des panoplies militaires, l'imbrication des rivalités et des haines. "Quand la fatalité crève sur un point du globe, elle l'encombre d'uniformes", écrivait Bernanos! Il aurait pu tout aussi bien dire : elle l'encombre d'armements. Hélas, la fatalité et le Sud ne cessent d'avoir partie liée.

- Pour rompre la dialectique conflits-armements, élever les seuils de défiance et d'agressivité et lutter au passage contre la psychose Nord-Sud, le meilleur moyen est d'agir sur les armes en les limitant, les contrôlant ou les bannissant. Après d'innombrables démarches infructueuses dans ce sens dont émerge, il est vrai, le traité de non-prolifération, celui de 1972 sur les armes bactériologiques et quelques ententes partielles, c'est l'objet des tous récents plans Bush et Mitterrand que la détente, l'affaire du Koweït, le regain du Conseil de Sécurité et l'inquiétude légitime que soulève la vulgarisation des progrès technologiques, justifient. Outre qu'il est plus complet que l'autre, le projet français a toutefois le mérite, non seulement de viser des objectifs catégoriels de réduction, d'interdiction ou de non-prolifération, mais d'évoquer les spécificités régionales qui doivent leur servir de cadre. On pourrait dire, une fois de plus, que la France est plus third world minded que l'Amérique, deux obstacles majeurs se présentant, celui de l'éga1ité de traitement des états et celui de leur souveraineté.

Sur le premier point, il est hasardeux, par exemple, de prétendre pousser les grands fournisseurs d'armes à réduire leurs ventes dans une perspective de limitation fort souhaitable au Proche-Orient, tout en annonçant d'importantes livraisons et $d$ 'importants financements militaires en faveur $d$ Israël, au moment même, $d$ ailleurs, où ce pays se durcit face aux propositions Baker. Deux poids, deux mesures que l'on risque de retrouver aussi sur le plan nucléaire. Certes, la sécurité d'Israël est essentielle. Mais comment pousser les voisins à désarmer si leur sécurité n'est pas au même titre assurée! Et comment, en agissant ainsi, vouloir être pris pour arbitre impartial! La manoeuvre d'arms control, si elle est globale, doit aussi s'adapter au contexte régional, en associant, d'abord, toutes les parties prenantes, et en tenant soigneusement compte, ensuite, des alliances, complémentarités, oppositions et clivages. Ce n'est pas vrai qu'au Proche-Orient. Les militaires argentins ont, le cas échéant, raison de s'étonner des pressions dont ils sont l'objet pour abandonner la fusée Condor tandis que le Général Pinochet semble trouver en Europe un accueil plutôt favorable à son projet de missile à courte portée.

Reste le problème de la souveraineté. On ne saurait dire que les grandes puissances, qui ont toujours exercé une espèce de pouvoir régulateur à travers le monde, ont tort de vouloir s'attaquer à la course aux armements et à la prolifération des armes de destruction massive ou déstabilisantes. Aujourd'hui où la situation Est-Ouest le permet, elles peuvent même se poser en exemples, tout en soulignant qu'elles participent, par la réduction de leur propre commerce des armes, au sacrifice général. On mesure, cependant, les obstacles, accrus du fait qu'il s'agit d'initiatives venant précisément du Nord et de nantis, au lendemain de la guerre du Golfe où, qu'on le veuille ou non, la précipitation américaine à rétablir la loi et l'ordre sans concession d'aucune sorte, a fait froncer les sourcils de bien des pays du tiers monde. Interdire, imposer, contrôler voilà qui n'est pas très conforme à un droit international qui décidément paraît n'être respecté par ceux qui l'ont fait que lorsqu'ils y trouvent leur compte : on comprend les réticences, face à une sorte de devoir d'ingérence que le Nord tend à s'arroger, face à un "nouvel ordre international" dont on a tout lieu de craindre la partialité et les déviances9. La pax americana pourrait finalement être comprise ainsi : chacun doit 
accepter le rang qu'on lui désigne dans son aire géographique en refoulant à l'occasion ses ambitions et en se contentant d'être un facteur d'équilibre ou un rouage dans une mécanique d'ensemble. Autant dire que pour qu'un état du Sud tire son épingle du jeu, il lui faut être du bon côté, être en quelque sorte un état relais - tantôt fondé de pouvoir et tantôt gendarme - tel que les Grands ont su en créer à leur dévotion. Dévotion relative, car la plupart des satellites de ce type n'ont pas manqué d'échapper assez vite à leur protecteur! Ainsi Israël, la Syrie, l'Irak, l'Iran, le Vietnam, l'Afrique du Sud qui ont mené sans vergogne leur propre jeu, qu'il se place dans une perspective expansionniste (invasion du Liban, du Cambodge ou du Koweï), dans une perspective de sauvegarde de l'ordre public international (réacteur d'Osirak, bombardement du QG de l'OLP à Tunis) ou même à une ingérence pure et simple dans les affaires du voisin (Liban depuis 1974, Angola, Mozambique, etc.). Si l'existence de ces amis-relais donne le sentiment que le Nord s'immisce partout, interfère dans l'économie, joue les redresseurs de tort ou les policiers, en fait, sur le plan des interventions militaires à proprement parler, la réalité est bien plus réduite. Les opérations significatives au Sud, non seulement ne sont pas nombreuses, mais sont rarement réussies. Pour l'être, elles supposent un problème en quelque sorte unique (Malouines, Zaïre, Panama) et échouent au contraire dès que le cadre est complexe (Liban, Afghanistan) non sans attiser au passage les antagonismes. A la limite, ce sont moins les "interventions" du Nord que les "non interventions" qui sont ici ou là regrettées : au Liban, au Cambodge, lieux de massacres collectifs, dans les territoires occupés, au Liberia, au Kurdistan gazé, dans les cas de famine enfin comme en éthiopie ou au Soudan.

- Le coût de plus en plus prohibitif des engagements au Sud, compte tenu de l'accroissement du niveau technologique des matériels utilisés fait, d'ailleurs, que très peu de pays peuvent y faire face. La guerre du Golfe est exemplaire en cela : il a fallu une coalition autour d'une puissance majeure pour écraser l'Irak. Conjoncture particulière, voulue ou provoquée, la crise apporte deux enseignements aux états sudistes émules de l'Irak: persuadez l'autre de votre innocence (personne n'a cru ou n'a voulu croire que) et étayez celle-ci avec un arsenal nucléaire capable d'interdire toute intervention. La dissuasion nucléaire a donc de beaux jours devant elle. Encore les règles du fort au fort ou du faible au fort établies jusqu'ici, risquent-elles de ne plus totalement jouer dans le nouveau contexte. Peut-on pour autant parler de dissuasion du fort au faible? Le faible se défend, telle la France face à l'URSS, mais se garde de provoquer plus fort que lui. S'il s'aventure comme l'Irak dans des voies condamnables, on ne brandira pas a priori à son encontre la menace nucléaire, usant plutôt de moyens coercitifs pour le faire reculer. Car, ne l'oublions pas, seuls les intérêts vitaux sont justiciables de l'arme atomique. Il est vrai que l'on peut avoir à faire à des comportements irrationnels qu'il faut être capable d'affronter; de même dans le morcellement et les rivalités régionales, à un jeu de dissuasions croisées qu'il convient d'empêcher de dégénérer. Comment? C'est là qu'on retrouve la contradiction relevée plus haut entre l'intérêt des pays nucléaires au maintien des règles de la dissuasion par le contrôle et l'interdiction, et la perception de ces mesures par le sud comme expression d'un nouvel impérialisme qui allierait plus que jamais domination économique et technologique avec puissance militaire. Le fond du problème, quoiqu'il en soit, est que la structure ancienne ayant éclaté, il n'y a plus, comme sur l'axe EstOuest, de dissuasion "existentielle", la crédibilité devenant question de circonstance et de cas particulier. C'est dire que le concept de la dissuasion du fort au faible, ici ou là 
évoqué, reste à explorer. L'autre versant c'est que ce n'est pas dans une logique NordSud que cette réflexion doit trouver son ancrage, mais bien dans la perception d'un commun intérêt entre pays voués à être les victimes potentielles, proches ou lointaines - eu égard aux portées des fusées balistiques - de voisins dangereux.

4 - L'engagement armé n'est qu'une superstructure. L'infrastructure, ce sont les tensions et affrontements politiques, économiques, sociaux ou culturels, dont les conflits sont non seulement le reflet, mais souvent la source, tant les perturbations, les risques, les menaces, les réactions qu'ils provoquent font du remue-ménage et bousculent des intérêts.

Conflits "enchevêtrés", "par contagion", ou à tiroirs : un litige banal dégénère, entre en résonance avec d'autres, embrase une zone, voire se propage au-delà. A ce titre, la distinction traditionnelle entre conflit local, régional et international, si elle correspond fréquemment aux réalités, est parfois factice. De même, la dichotomie qu'on a tendance à établir à propos du rôle joué, au temps de la bipolarité, par les grandes puissances dans les luttes armées du Sud, les uns le majorant et ne voyant dans celles-ci qu'un exutoire à la dissuasion nucléaire centrale, les autres récusant au contraire la géopolitique pour ne retenir que la sociologie ou l'anthropologie domestique.

Est-on, à cet égard, conduit à voir la conflictualité dépérir (ou tout au moins diminuer) dans les tiers-monde, compte tenu de l'effondrement du communisme et de l'Union soviétique, ou enfonce-t-elle ses racines trop profondément pour qu'il en aille autrement ${ }^{10}$ ? D'un côté, nombre d'affrontements sont nés, se sont développés ou se sont prolongés du fait de l'antagonisme Est/Ouest, le Sud étant transformé en terrain de parcours où les Grands se battaient par personnes interposées. Là cependant où l'enjeu, et par la suite la zone (ou la zone puis l'enjeu) était stratégique comme en Afrique du Sud, dans la Corne, au Proche-Orient ou dans le Sud-Est asiatique, la querelle tendait à prendre une dimension majeure, homothétique de la querelle planétaire. Ceci n'a plus la même raison d'être, pas plus que n'ont de raisons de se perpétuer nombre d'affrontements idéologiques désormais frappés d'archaïsme qui justifiaient l'intervention hostile ou complice des Grands. N'assiste-t-on pas, d'ailleurs, à une récession très nette des conflits! 38 en 1986, 33 en 89, puis 31 en 1990 selon le SIPRI. Mieux, si l'on prend un exemple concret, l'Afrique australe, on constate non seulement que la fin de l'affrontement Est-Ouest y dévalorise les matières premières stratégiques mais que dans son ensemble elle perd un certain poids : départ des Cubains, mise hors circuit partielle des forces de l'ANC reconversion en cours de l'armée sud-africaine accompagnée d'une baisse de 11 à $15 \%$ du budget militaire, reconversion de la marine de haute mer en garde-côtes, enfin, appel à la démilitarisation de la zone Sud-Ouest de l'océan Indien récemment lancé par la commission internationale censée avoir cette zone en charge Tout cela montre bien dans quel sens va la tendance !

S'ajoute que la faillite idéologique soviétique s'est conjuguée avec la faillite économique. Pour le continent africain les années 1983-1991 sont les années noires de la décroissance, des PNß négatifs, de l'énormité de la dette, de la dévastation des campagnes ici par le collectivisme et là par les guerres, tous faits que l'on peut appliquer aussi bien à l'Amérique du Sud qu'aux pays maghrébins. Bref, il est clair que le poids militaire de ces pays s'est considérablement amenuisé et que le freinage de la spirale du sous-développement a pris le dessus sur les envies de grandeur militaire ou de puissance. Sans doute peut-on se demander si la détresse économique est susceptible 
de conduire à une fuite en avant, notamment à l'invasion d'un pays voisin? Mais, en fait, en éthiopie comme au Soudan, on constate que la famine est souvent le corollaire de l'effondrement des structures étatiques, lesquelles sont seules à même de mobiliser les populations à des fins extérieures. On chassera plutôt les étrangers, comme au Nigeria en 1985, ou en Mauritanie, quatre ans après. En sens inverse, il va de soi que le carcan bipolaire avait le mérite de geler certaines situations que rien n'empêchera plus aujourd'hui de dégénérer, soit que le désordre et l'anarchie s'accroissent, soit que les appétits et les capacités de diverses puissances régionales grandissent, soit que dans un cadre multipolarisé, sinon multipolaire, les intérêts croisés, de quelque nature qu'ils soient, s'opposent sans frein.

- Les conflits marqués par la bipolarité sont réduits à l'état de vestiges. Les conflits purement locaux, échappent par leur nombre et leur incohérence à l'analyse et à la rationalité : luttes ethniques, religieuses ou frontalières, affrontements de sédentaires et de nomades, de blancos et de colorados, de forestiers et de côtiers, de privilégiés et de miséreux ils attisent, certes, l'effervescence générale, mais constituent un arrière fond de violence sans portée internationale véritable, tout au plus justiciables qu'ils sont du coup de patte d'un voisin ou d'une intervention humanitaire justifiée par la détresse de millions de réfugiés. C'est à l'échelon au-dessus qu'il faut se placer, là où les dimensions Sud-Sud et Nord-Sud apparaissent et le cas échéant s'entremêlent, où, audelà du cadre géographique impliqué, des prises de conscience internationales et des réactions hostiles ou complices se révèlent: réactions du Nord notamment, ramifications géopolitiques qui, plus largement, surdimensionnent le débat. A l'heure de l'interdépendance et des médias, on ne saurait, à cet égard, s'en tenir à la pure sociologie ou à l'ethnologie: la polémologie moderne exige la prise en compte des interFérences multiples dont la conflictualité se hérisse désormais.

Distinguons ici, pour simplifier, deux niveaux : celui des équilibres ou déséquilibres régionaux, de plus en plus dominés par les flux Sud-Sud, et le niveau où trop d'intérêts sont en cause, trop de dangers - nucléaires en particulier - se profilent pour que le Nord reste l'arme au pied.

Déséquilibres régionaux pour commencer, que plusieurs exemples peuvent, entre autres, illustrer. Cas du chaos libérien tel que toute la région s'en trouve affectée, sans que toutefois s'en mêle le Nord, découragé par un désordre sans fin. L'abcès de fixation est de taille, mais sa portée se trouve du même coup limitée. La Libye et l'Irak fournissent aux différentes factions leurs armes et par là soufflent sur le foyer. La CDEAO éclate, les francophones refusent dans leur majorité de suivre les anglophones menés par le Nigeria dont la puissance et les ambitions régionales crèvent l'écran. "Ecomog", sans plan de guerre, et sans même disposer de cartes pour débarquer brille par son impréparation et, dans une certaine mesure, sa partialité, mais réussit plus ou moins à s'imposer, premier exemple d'une force régionale spontanée qu'il faut saluer, même si elle revêt les traits anarchiques du conflit qu'elle prétend traiter. La France s'en tient à l'aide humanitaire; les Etats-Unis hésitent à s'engager, se contentant d'assurer une certaine logistique et de fournir quelques complicités. Situation implosive ingérable qu'aucune stratégie véritable ne vient ordonner.

Les conflits de la Corne d'Afrique s'ouvrent sur un paysage plus large. Pour un temps, le contexte Est-Ouest domine, mais est très vite obscurci par mille chassés-croisés: abandon par l'URSS de la Somalie au profit de l'éthiopie et des mouvements marxistes 
érythréens, soutien de Mogadiscio par les Etats-Unis, appuis islamistes contre appui israélien, imbrication des irrédentismes illustrés par la terre de l'Ogaden et le soulèvement tigréen, interférences des luttes civiles et religieuses du Soudan où l'on retrouve parallèlement les mêmes visées et les mêmes influences aggravées du jour où le pétrole se met à couler. Même impuissance du Nord à rationaliser et à régler un affrontement qu'il a au départ plus ou moins attisé et que la famine vient dramatiser. Bref, conflit enchevêtré à l'extrême imprudemment exacerbé de tous côtés, lieu géométrique de tant de contradictions et de haines, que nul ne paraît aujourd'hui en mesure de régenter.

Dans la même ligne, on pourrait évoquer, même si ses effets sont plus dilués, la poussée arabo-musulmane en direction de l'Afrique noire. Reprenant les vieilles voies de pénétration datant du XIIème siècle, cet expansionnisme adopte, on le sait, plusieurs formes : la forme agressive longtemps privilégiée par Kadhafi, avec sa légion islamique et son grand rêve d'empire des sables ; la voie du clientélisme par vente d'armes, suivie notamment par l'Irak au Soudan, en Ethiopie et en Mauritanie; celle du prosélytisme religieux doublé d'une aide financière plus ou moins conditionnelle, chère à l'Arabie Saoudite et à divers émirats, cette tactique étant, le cas échéant, complétée par la conversion à l'Islam de quelques chef d'état; monopolisation enfin de l'OUA par des problèmes du Moyen-Orient étrangers aux vrais problèmes du continent autant de menées qui non seulement provoquent des réactions, comme le projet zaïrois de Ligue des états d'Afrique noire, visant à faire pièce à la Ligue arabe, mais qui attisent les rivalités comme la discorde Mauritanie-Sénégal a pu amplement le montrer.

Stratégie diffuse, spontanée, à laquelle on peut opposer d'autres stratégies plus volontaristes, et plus ordonnées : ainsi, la stratégie maritime de New Delhi dans l'océan

Indien ${ }^{11}$, qui, dans une optique de non alignement pacifique, vise à faire de cet océan un "lac de paix" pour le purger de la présence étrangère et y instaurer une mainmise que sa position géographique, son développement maritime, son poids démographique, sa puissance militaire, et sa diaspora, lui suggèrent. Pour des raisons internes et externes, l'Inde, certes, n'est pas au bout de ses peines.

L'Occident, fort du principe de la liberté des mers et de sa supériorité séculaire sur ce plan, n'est pas prêt de se laisser écarter. Mais, en attendant que la Chine fasse de même dans son aire, voilà une puissance du Sud qui d'ores et déjà prend rang et entend à l'avenir rivaliser.

$\mathrm{Au}$ moins la pression indienne reste-t-elle régionale et, d'une certaine manière, théorique. Les intérêts du Nord ne sont, en l'occurrence, qu'indirectement mis en cause, et l'on ne peut jusqu'à nouvel ordre parler de danger. Il n'en a pas été de même dans le Golfe où, à l'évidence, les Etats-Unis ont jugé, ou feint de croire, que l'entreprise de Saddam Hussein menaçait précisément leurs intérêts et constituait, pour la région en général et pour Israël en particulier, un risque inacceptable. S'agit-il d'une situation tout à fait spécifique ou la chose peut-elle ailleurs se reproduire? Nulle région au monde n'est, à vrai dire, à ce point polémogène et ne présente autant de fractures et de ruptures aussi prononcées: velléités d'hégémonie sur une zone éminemment stratégique, menaces sur le plus grand flux d'approvisionnement et les plus grandes réserves de pétrole, mise en cause éventuelle de l'état juif, conjonctions explosives d'idéologies agressives et sources inextricables d'antagonismes, capacités financières d'acquérir un armement sophistiqué et de tenir sous son feu les points les plus sensibles du globe, aussi bien terrestres que maritimes la liste est sans équivalent! 
I1 reste qu'ailleurs des circonstances similaires peuvent survenir qui attentent à la liberté d'action du Nord, violent les grands principes, lèsent ce qu'il considère comme ses intérêts, bouleversent les équilibres, lui donnent le sentiment d'être devenu vulnérable du fait notamment de la précision des fusées, bref, le conduisent à intervenir pour l'une ou l'autre de ces raisons en réplique à une agression ou de façon plus ou moins préventive. Ce peut être le cas, en particulier, en Méditerranée et dans le Sud-Est asiatique où les pesanteurs locales et les différentiels avec le Nord sont tout spécialement marqués, mais ce peut être le cas aussi dans bien d'autres régions, au fur et à mesure que le développement permettra à tel ou tel état sudiste de s'affirmer, en exprimant ses ambitions et sa volonté de puissance à l'image de ce qu'il nous a toujours paru naturel que le monde septentrional fasse et de ce qu'il a effectivement toujours fait. A ce titre, il faut bien se dire qu'il ne s'agit pas - si menace il y a - d'une menace Sud-Nord, mais d'une menace existentiellement réciproque qui appelle moins l'établissement d'un "ordre" que d'équilibres collectifs auxquels chacun doit être associé.

Du grand désordre en partie figé ou filtré par la bipolarisation, on risque, en définitive, de passer à un désordre effervescent, accentué ici par la faillite économique et l'augmentation des inégalités, là, par la résurgence de l'Islam et des nationalismes, ailleurs par le trop-plein démographique et sa tendance à s'exporter, enfin par l'émergence de pôles solidement armés, décidés à faire valoir leurs droits en piétinant au besoin ceux des voisins, en tout cas à s'imposer.

Anarchie et multipolarité, comment empêcher, d'un côté, le déferlement du terrorisme, les migrations sauvages, le narco-trafic et la radicalisation intégriste, et de l'autre le risque que la prolifération des armes à haute technologie et la croissance des appareils conventionnels font courir. Le développement est un premier levier qui semble, en Asie par exemple, apporter la stabilité. Outre, cependant, que c'est une longue patience, il peut tout aussi bien, comme l'Irak l'a prouvé, pousser à des ambitions démesurées. Seconde solution, à l'heure où l'on parle tant de Force d'Action Rapide: jouer les gendarmes en se tenant prêt à châtier, comme dans le Golfe, les "coupables" au premier dérapage. C'est d'action en réaction et de méfiance en suspicion militariser, toutefois, le monde encore plus qu'il ne l'est. Pire, c'est le dérégler en sapant les quelques règles qui l'encadrent si l'on invoque, pour être plus efficace, le "droit d'ingérence", fut-il transformé en devoir par souci de moralité.

L'unicentrisme temporaire tel qu'il ressort, par ailleurs, du triomphe de l'Amérique au Koweït s'il peut, de son côté, permettre de vider quelques abcès et de faire converger quelques intérêts opposés risque fort - arme à double tranchant - d'indisposer plus que de rassurer, en faisant passer pour intéressées, ethnocentriques et impérialistes les mesures les plus fondées. "Nouvel ordre international", non pas. Nouvelle coopération à géométrie variable selon les régions, visant à combler partiellement les fossés et à faire prendre conscience que le gonflement des arsenaux et le recours aux armes est la pire des façons de régler le différend Nord-Sud, axe fondamental désormais de relations internationales trop longtemps accaparées par une lutte idéologique aujourd'hui dépassée. 


\section{NOTES}

1. Gulliver empêtré, Interfutur, Le Monde rompu.

2. The National Interest, no 20, été 1990.

3. Le cycle de la dissuasion, Paris, La découverte/FEDN, 1990, p. 286 et suivantes.

4. Bertrand Warusfel, "Les risques de prolifération vers certains pays tiers", Les fondements de la sécurité et de la paix en Europe à l'horizon 2000, SGDN, septembre 1990.

5. Philippe Richard, "L'arme nucléaire restera-t-elle le privilège des puissants ?, Le Monde Diplomatique, janvier 1991.

6. Jean-Marc Balencie, "Le développement des forces navale dans le tiers monde", Stratégique, 3/1989.

7. Construction de son premier sous-marin d'attaque classique en 1992.

8. Controlling the arms trade as a contribution

9. Philippe Moreau Desfarges, Le devoir d'ingérence, RDN, juin 1991.

10. Sharam Chubin, "Les conflits dans le tiers monde, Revue internationales de sciences sociales, nœ 127, février 1991.

11. Jean Alphonse Bernard, "L'Inde, puissance navale, Stratégique, nœ 48, 4ème

trimestre 1990.

\section{RÉSUMÉS}

Les auteurs se proposent d'étudier la géopolitique des armements : si certains pays (en Afrique subsaharienne) ne sont presque pas armés, d'autres possèdent des armes mais peu de moyens de les utiliser. Cependant, quelques pôles de pouvoir émergent : par exemple, l'Inde, la Chine, l'Iran, et le Brésil. Cet équilibre instable et ses conséquences seront étudiés dans la seconde partie : les difficultés et les effets pervers du contrôle du marché de l'armement ainsi que le coût élevé des interventions dans le Sud (la Guerre du Golfe). Cette situation entraîne deux types de risques : d'un côté, le terrorisme, les migrations incontrôlées et le trafic de stupéfiants ; de l'autre, le risque de prolifération des armes à haute technologie et la croissance des engins de guerre conventionnels. Dans un tel contexte, seul un nouveau type de coopération aiderait à faire comprendre qu'un recours aux armes est la pire solution pour régler les différends Nord/Sud.

In a first section, the authors draw a geopolitical sketch of world armaments : if some countries (in sub-Saharan Africa) are almost defenceless, others do have weapons but with a low ability to use them ; they also point out the emergence of a few poles of military power such as India, China, Iran, Iraq and Brazil. This unstable equilibrium and its effects are studied in a second section : difficulties and perverse effects of supervising the arms trade, high costs for intervention in the South (the Gulf War). A situation which leads to two kinds of risks : on one side terrorism, uncontrolled migrations or narco-traffics, on the other, a risk of proliferation of high technology weapons and the growth of conventional military machineries. In such a case, 
only a new type of co-operation might help to make clear that having recourse to arms is the worst way to settle the disagreements between North and South.

INDEX

Index géographique : Pays du Sud (Tiers Monde)

Mots-clés : armes et armement, Militaires, menace

\section{AUTEUR}

\section{MARIE-LUCY DUMAS}

Professeur à Paris I, agrégé de droit public et sciences publiques, Président de la FEDN et Professeur agrégée d'histoire-géographie. 